\title{
Elaboración de una Pasta Emulsionada de Cáscara de Chontaduro (Bactris gasipaes)
}

\section{Preparation of an Emulsified Paste of Peach Palm Peel (Bactris gasipaes)}

\author{
Juan Felipe González Henao ${ }^{1}$ \\ Gonzalo Gnecco Mancheno ${ }^{1}$ \\ Aybi Patiño ${ }^{1}$ \\ Eduardo Enciso Peña ${ }^{1}$ \\ María Isabel Páez ${ }^{1}$ \\ ${ }^{1}$ Corporación Biotec, Km 17 Recta Cali-Palmira, Colombia \\ juan1992felipelgmail.com \\ necco69ehotmail.com \\ aybipatiegmail.com \\ eeelcorporacionbiotec.org \\ maria.paez.valencialoutlook.com
}

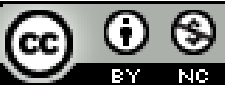

Enviado:

$2020 / 12 / 20$

Aceptado:

$2021 / 01 / 28$

Esta obra está bajo una licencia internacional

Publicado:

$2021 / 06 / 30$

Creative Commons Atribución-NoComercial 4.0.

\section{Resumen}

El Chontaduro es una fruta exótica tropical típica de las selvas del sur y suroccidente de Colombia, con arraigo ancestral en las comunidades de estas regiones. Proveniente de la palma de chonta, es consumido tradicionalmente fresco y cocinado. Actualmente, se producen alrededor de 44 Ton/año de cáscaras de chontaduro en la región del Cauca, Valle del Cauca y Putumayo; cada una de estas aporta una tercera parte de esa producción. Algunas industrias han empezado a desarrollar su consumo masivo, procesando de formas novedosas la pulpa de esta. Durante la operación de pelado, estas industrias generan un coproducto utilizando el proceso denominado cáscara con adherencia de pulpa. El objetivo de este trabajo fue agregarle valor a este coproducto elaborando una pasta emulsionada que sirve como ingrediente para la elaboración de alimentos en los que el chontaduro es la característica organoléptica principal. Además, se describen las condiciones de operación encontradas mediante experimentación a nivel de laboratorio y se establecen valores cuantitativos para dichas condiciones. También, se realizaron los análisis de composición (tabla nutricional), fisicoquímicos, microbiológicos y vida útil. Estos valores quedan disponibles para realizar un escalamiento del proceso a escala industrial. Las pruebas de concepto del producto muestran interesantes características potenciales; abre una nueva línea derivada del chontaduro: una pasta que facilita la adición de un ingrediente estable, que remite al sabor del chontaduro en platos o

Sumario: 1 Introducción, 2 Materiales y Métodos, 3 Resultados y Discusión, 4 Conclusiones.

Como citar: González, J., Gnecco, G., Patiño, A., Enciso, P., \& Páez, M. (2021). Elaboración de una Pasta Emulsionada de Cáscara de Chontaduro (Bactris gasipaes). Revista Tecnológica - Espol, 33(1) 72-83.

http://www.rte.espol.edu.ec/index.php/tecnologica/article/view/794 
formulaciones de alimentos con la comodidad de poderse hacer en cualquier momento del año, con disponibilidad inmediata y sin necesidad de un procesamiento del fruto.

Palabras clave: Bactris gasipaes, biomasa residual, cáscara de chontaduro, emulsión, ingrediente natural, pasta de chontaduro.

\begin{abstract}
Peach palm is a typical tropical exotic fruit of the jungles in the South and Southwest of Colombia, with regional ancestral roots in the communities. It is traditionally consumed fresh or cooked, coming from La Palma de Chonta. Annually, 44 tons of peach palm peels are generated in the Cauca, Valle del Cauca, and Putumayo regions. Each region contributes a third of that production. Some industries have begun to develop by their mass consumption, creating novel ways of presenting their pulp. These industries generate a by-product in their process called peel with pulp adhesion, during the peeling operation. The objective of this work is to add value to this product by making an emulsified paste that serves as an ingredient for food preparation in which peach palm is the organoleptic characteristic. The operating conditions found by laboratory experimentation are described, and quantitative values are established for these conditions. Composition (nutritional table), physicochemical, microbiological, and shelf-life analyses were carried out and these values are used to scale the process to industrial size. The product concept tests show interesting potential characteristics, it opens a new line derived from peach palm, a paste that facilitates the addition of a stable ingredient, which refers to the flavour of peach palm in dishes or food formulations. This creates the convenience of production year-round, with immediate availability and no need for fruit processing.
\end{abstract}

Keywords: Bactris gasipaes, peach palm paste, peach palm peel, emulsion, natural ingredient, residual biomass.

\title{
1. Introducción
}

El Chontaduro es un fruto de la palma de chonta, tradicionalmente consumido por las poblaciones de las zonas de la selva húmeda tropical de Colombia, especialmente, en la costa Pacífica, selva del Amazonas y algunas regiones selváticas del Orinoco (Reyes et al., 2002).

Es un cultivo artesanal, con una cadena de valor poco estructurada y serios problemas de plagas en el cultivo por desequilibrios ecológicos. Produce dos cosechas abundantes al año. El procesamiento (lavado, cocción y pelado) está distribuido geográficamente en personas que lo procesan y el consumo es disperso debido a la itinerancia de los vendedores (Hernández et al., 2008). Unas pocas fábricas en Cali (Valle), El Tambo (Cauca) y Villa Garzón (Putumayo) procesan la fruta del chontaduro para darle mayor valor agregado y generar productos derivados (Giraldo et al., 1999).

Dos fuerzas dirigentes motivan el desarrollo del producto analizado en este artículo: a) el aprovechamiento de una biomasa residual, que actualmente no tiene un uso en las empresas que dan valor agregado al chontaduro b) la búsqueda de un producto accesible como fuente potenciadora del sabor de esta fruta, propia de las regiones selváticas de Colombia e ingrediente en la cocina artesanal de restaurantes o en las formulaciones de productos industriales (López-Calvo et al., 2015).

Entre los estudios precedentes que involucran productos derivados del chontaduro, se encuentran aquellos que determinan la concentración de pigmentos carotenoides en harina de residuos de chontaduro y que indican el aprovechamiento de subproductos. Estos se generan luego del consumo del chontaduro, al evaluar la harina residual de cáscara de este fruto a partir de la identificación y cuantificación de carotenoides; para esto se utiliza una columna YMC en un equipo HPLC con longitud de onda fija en $450 \mathrm{~nm}$. Sus resultados evidencian la presencia de violaxantina, luteína, zeaxantina, variedad de beta carotenos y un alfa caroteno, lo que comprueba la importante presencia de pigmento carotenoide y provitamina A: lo que representa un gran aporte para la industria alimentaria (Martínez y Ordónez, 2016). 
En el mismo sentido, existen investigaciones del subproducto del chontaduro (harina fabricada de las cáscaras de esta fruta) donde se analiza la composición fisicoquímica de esta harina por secado convectivo (Martínez-Girón et al., 2017), que han determinado la presencia de variados compuestos bioactivos: carotenoides, compuestos fenólicos y otros. Además de esto, se evalúa el encapsulado de betacarotenos extraído a partir de estos residuos de chontaduro, en un estudio de estabilidad que usaron dos procesos de secado por atomización (Ordóñez-Santos et al., 2018). Y para finalizar con la línea de investigación, se ha realizado la extracción de carotenoides totales a partir de epicarpio o cáscara de chontaduro seca y molida mediante la aplicación de ultrasonido y aceite vegetal como agente extractor (Girón et al., 2019).

Se incluyen dentro de los antecedentes, publicaciones del aprovechamiento de residuos o subproductos del pelado de chontaduro provenientes de Perú, Ecuador, Costa Rica y departamentos colombianos, como los de Cauca, Putumayo y Amazonas (Sam y Vásquez, 2002). También la caracterización del fruto en partes, como el mesocarpio o pulpa, epicarpio o cáscara, determinan en su composición factores antinutricionales y nutrientes principales (Restrepo y Estupiñán, 2011). Su uso es tan variado como su forma de harina, por ejemplo, para un producto fermentado a base de subproductos del chontaduro, tanto para consumo animal como en seres humanos (Rojas et al., 2011; Murillo et al., 2016). Cabe aclarar que no se han encontrado iniciativas con este subproducto del chontaduro para crear una pasta a nivel de prototipo o con posibilidad de escalamiento a nivel industrial.

El propósito de este documento es el de describir la manera en cómo se obtuvo el prototipo de un producto derivado de las cáscaras del chontaduro denominado 'pasta emulsionada de cáscara y pulpa de chontaduro' para uso en el sector alimenticio.

\section{Materiales}

\section{Materiales y Métodos}

1) Chontaduros: se usaron chontaduros maduros crudos, obtenidos en el centro de acopio comercial de por estos frutos denominado Puerto Chontaduro, en Cali, Colombia; Calle 34N \# 3N24; adquiridos en racimos.

2) Agua: se usó agua destilada obtenida en un laboratorio por evaporación y condensación.

3) Aditivos: se usaron aditivos de grado alimenticio comprados comercialmente en un distribuidor. Son de origen natural o petroquímico.

\section{Métodos para medición de aguas residuales}

Se usaron los métodos analíticos contenidos en el Standard Methods for the Examination of Water and Wastewater; 23.a Edición, 2018 (Ministerio de Ambiente y Desarrollo Sostenible, 2010; Ministerio de Ambiente y Desarrollo Sostenible, 2015).

\section{Microbiología}

Se usaron los métodos analíticos de determinación para cada tipo de microorganismos; se hizo, por siembra en una placa de agar nutritivo específico para cada microorganismo (Ministerio De Salud y Protección Social, 2013; FAO/WHO, 2019), cultivo en una incubadora y conteo de colonias (Levaduras BIO-PR-158 V3 (2016-08-25); Bacterias Mesófilas Aeróbicas: Método NTC 4519 (200904-15); Mohos BIO-PR-158 V3 (2016-08-25); Escherichia Coli: NTC 4458 (2007-12-12); Salmonella: ISO 6579-1:2017; Coliformes fecales y totales: NTC 4458 (2007-12-12); Estafilococo coagulasa: NTC 4779 (2007-08-29); Esporas Clostridium: ICMSF (Comisión Internacional de Especificaciones Microbiológicas para los Alimentos). INVIMA:1998. Cap.2, Num.10; Bacillus Cereus: NTC 4679 (2006-08-30).

\section{Plaguicidas}

Se hace por la metodología de cromatografía de gases acoplada a espectrometría de masas sobre una solución hecha con los materiales macerados con hexano. Se sigue métodos de residuos múltiples por GC-MS/MS y LC-MS/MS para más de 560 compuestos [18] para la cuantificación de pesticidas y cuantificación de glifosato, AMPA y glufosinato en frutas, verduras y cereales (Ministerio De Salud y 
Protección Social, 2013; FAO/WHO, 2019; Ministerio de Agricultura y Desarrollo Rural y de la Protección Social, 2007).

\section{Análisis proximal y tabla nutricional}

Se usaron los métodos analíticos definidos por la legislación colombiana pertinente (normas ISO 1871, AOAC 985,35; 923,03; 985,29; 923.09, ICP-OES, BIO PT-019 V4) para la caracterización de composición de alimentos, incluyendo el cálculo por diferencia para la determinación de carbohidratos y calorías.

\section{Análisis fisicoquímicos}

Humedad y materia volátil (pérdida por secado a $103^{\circ} \mathrm{C}$ ), acidez (expresado como ácido oleico) volumetría a pH 8,3, índice de peróxidos (NTC 236), residuos de ignición (basado en AOAC 923,03), viscosidad Brookfield DV1-LV (viscosímetro Aguja No. 3; $5 \mathrm{rpm}$ a $35^{\circ} \mathrm{C}$ x $1 \mathrm{~min}$ ), densidad a $20^{\circ} \mathrm{C}$ (picnometría), tamaño de partícula (diámetro medio), difracción láser equipo Cilas 920, pH directo a $20,4^{\circ} \mathrm{C}$ (BIO-PT-036 determinación de $\mathrm{pH}$ por potenciometría), contenido de gluten (Inmunoensayo enzimático/basado en AOAC 2012.01), sólidos solubles ${ }^{\circ}$ Brix (refractometría), metales pesados por ICP-OES (espectrometría de masas de plasma o plasma de acoplamiento inductivo es una fuente de ionización que, junto a un espectrofotómetro de emisión óptico (OES) constituye el equipo de ICPOES) (Ministerio De Salud y Protección Social, 2013; FAO/WHO, 2019).

\section{Vida útil acelerada}

GTC 165: Análisis sensorial. Metodología. Guía General NTC 2680: Análisis Sensorial. Metodología. Prueba de Comparación Pareada. NTC 5328: Análisis Sensorial. Directrices para el uso de escalas de respuesta cuantitativas.

\section{Prueba de concepto}

Por medio de prueba hedónica adaptada de 7 puntos (Drake, 2007), recomendada por la mayoría de los estudios o proyectos de investigación estándar, se puede determinar si existen diferencias entre los productos en la aceptación del consumidor. La escala de 7 puntos asigna dicha puntuación desde la menor valoración ( $1=$ me disgusta mucho) hasta la máxima puntuación ( $7=$ me gusta mucho), pasando por el resto de los valores $(2=$ me disgusta moderadamente, $3=$ me disgusta poco, $4=$ no me gusta ni me disgusta, $5=$ me gusta poco, $6=$ me gusta moderadamente) .

A los 5 panelistas se les pide evaluar muestras de varios productos ( 3 en este caso), indicando cuánto les agrada cada muestra marcando una de las categorías en la escala. Cabe resaltar que la escala puede ser presentada gráfica, numérica o textualmente (Clark et al., 2009).

Las características que se evalúan son organolépticas (color, olor, textura, sabor), las cuales se dividen en una seria de categorías determinadas. Hay un espacio para comentarios adicionales, si es el caso, en cada categoría evaluada. Se pueden comparar los datos de consumo (escala hedónica) empleando en el análisis la prueba no paramétrica de Friedman combinado con el procedimiento Nemenyi (Bayarri et al., 2012).

\section{Características de la Cadena de Valor}

\section{Resultados y Discusión}

La Figura 1 muestra la cadena de valor del proceso de la pasta emulsionada de cascara y pulpa de chontaduro.

El proceso que se describe es parte de la cadena de valor del chontaduro, que empieza en el cultivo, sigue en las empresas que procesan al chontaduro y le agregan valor al convertirlo en productos directamente utilizables por el cliente, denominadas en la Figura 1, genéricamente, como industria primaria (Graefe et al., 2013). 
Dentro de esta cadena, el proceso de producción de la pasta emulsionada de cáscara y pulpa de chontaduro (PECPC) corresponde a uno más de los productos derivados del chontaduro y a una ampliación del portafolio de estas industrias agrupadas como industria primaria.

\section{Figura 1}

Cadena de valor de la pasta emulsionada de cáscara y pulpa de chontaduro

CADENA DE VALOR DEL PROCESO DE PRODUCCION DE PECPC

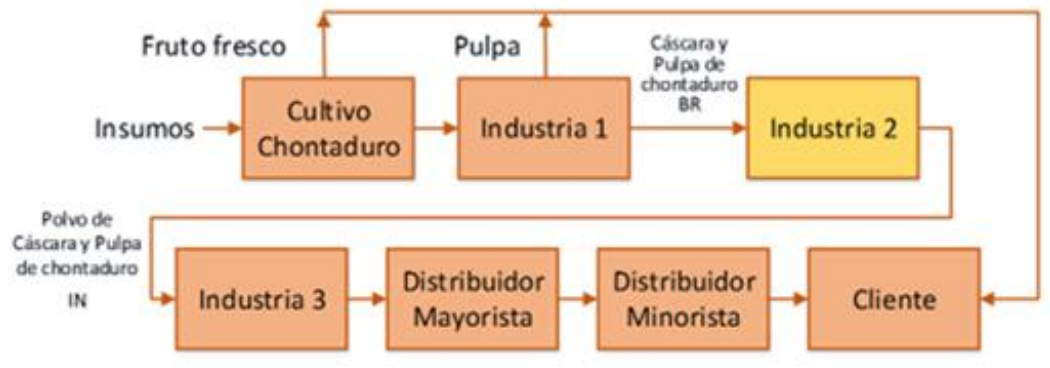

Fuente: Elaboración propia

Se ha denominado industria secundaria a aquella que procesará las cáscaras con pulpa adherida generadas en la industria primaria y que se describe en este artículo. La PECPC será usada por la industria final que fabricará los productos, que serán distribuidos por una cadena formada por un distribuidor mayorista y un distribuidor minorista hasta llegar al cliente o consumidor final. En esta cadena, los restaurantes artesanales y las empresas de suministro de comidas industrializadas (catering en inglés) no necesariamente tendrán que pasar a través de los canales de distribución.

Esta cadena puede tener acoplados sistemas logísticos de transporte y almacenamiento que se han omitido en aras de simplicidad. Este diagrama permite ubicar el proceso dentro de la complejidad que tiene la cadena de valor.

\section{Características de la Materia Prima}

El producto que genera el cultivo es un racimo de chontaduro con frutos maduros, y es la unidad que comercialmente se intercambia. La Figura 2 muestra la composición promedio de un racimo de chontaduro y el peso en porcentaje de un fruto de este. Un racimo puede pesar entre 5 y $12 \mathrm{Kg}$, y un fruto, entre 15 a 45 gramos (Valencia et al., 2013; MADR, 2017).

Figura 2

Composición de las partes del racimo y el fruto del chontaduro

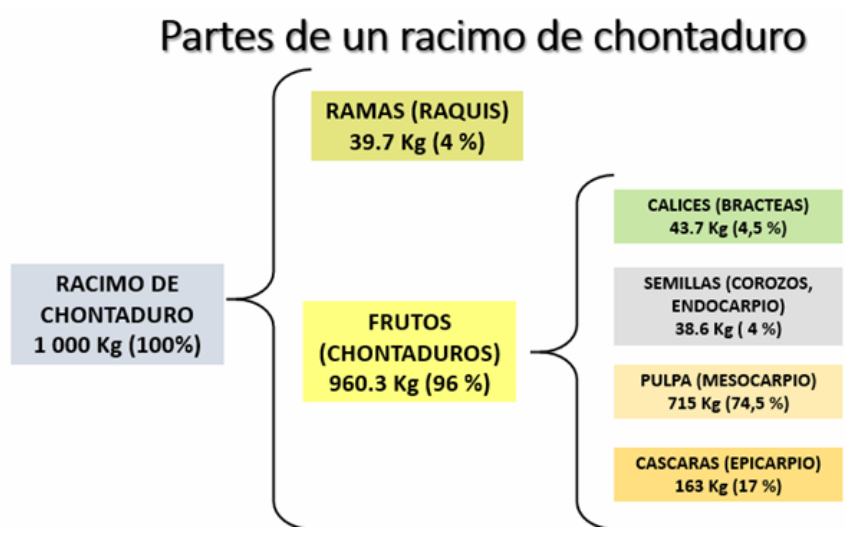

Fuente: Elaboración propia

La Figura 3 muestra la materia prima tal como llega desde el proveedor con sus características básicas. Esta materia prima, denominada cáscara con pulpa adherida, se origina al pelar manualmente los frutos de chontaduro lavados y cocinados en la industria primaria. 
Se encontró que la cáscara de chontaduro proveniente del pelado manual de la fruta contiene el $36 \%$ de material fibroso, correspondiente a la piel o cáscara (epicarpio), y $64 \%$, correspondiente a pulpa adherida (mesocarpio). Del total del fruto, esta cáscara con pulpa adherida corresponde al $16 \%$ de su peso total.

Figura 3

Cáscaras con pulpa adherida del pelado del chontaduro

BIOMASA RESIDUAL

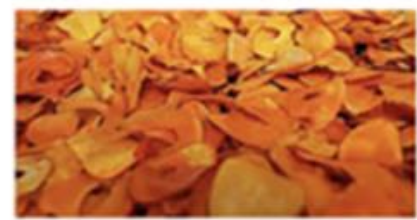

PARTES DE LA BIOMASA RESIDUAL

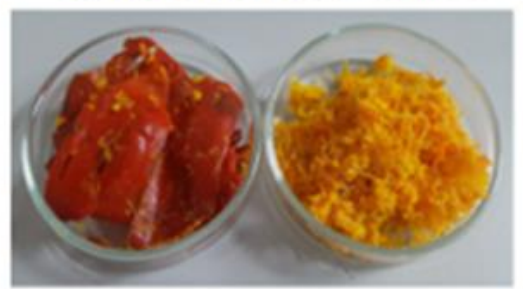

CÁSCARA

PULPA

(EPICARPIO)

(ENDOCARPIO)

El análisis proximal de la cáscara del chontaduro se muestra en la Tabla 1, en la que se compara con harinas de pulpa de chontaduro y con una harina de pulpa y cáscara de chontaduro.

\section{Tabla 1}

Composición de la cáscara con pulpa adherida de chontaduro, harinas de chontaduro comerciales y harina de solo cáscara

\begin{tabular}{|c|c|c|c|c|c|}
\hline Análisis & Unidad & $\begin{array}{c}\text { HC sin } \\
\text { Cáscara } \\
\text { promedio }^{1}\end{array}$ & $\begin{array}{c}\text { HC sin } \\
\text { Cáscara }(\mathrm{DE})^{2}\end{array}$ & $\begin{array}{l}\text { HC con } \\
\text { Cáscara }^{3}\end{array}$ & $\begin{array}{l}\text { Cáscara de } \\
\text { chontaduro }^{4}\end{array}$ \\
\hline Carbohidrato & $\mathrm{g} / 100 \mathrm{~g}$ & 78,46 & 4,33 & 72,08 & 39,84 \\
\hline Grasa total & $g / 100 \mathrm{~g}$ & 6,65 & 4,33 & 11,93 & 6,25 \\
\hline Proteína & $\mathrm{g} / 100 \mathrm{~g}$ & 5,37 & 0,03 & 4,92 & 3,98 \\
\hline Cenizas & $\mathrm{g} / 100 \mathrm{~g}$ & 1,90 & 0,01 & 1,71 & 0,81 \\
\hline Humedad & $\mathrm{g} / 100 \mathrm{~g}$ & 7,63 & 0,01 & 9,36 & 49,12 \\
\hline Total & $\mathrm{g} / 100 \mathrm{~g}$ & 100 & 100 & 100 & 100 \\
\hline Humedad & $\mathrm{g} / 100 \mathrm{~g}$ & 7,63 & 0,01 & 9,36 & 49,12 \\
\hline Sólidos secos & $\mathrm{g} / 100 \mathrm{~g}$ & 92,37 & 0,01 & 90,64 & 50,88 \\
\hline Total & $\mathrm{g} / 100 \mathrm{~g}$ & 100 & 100 & 100 & 100 \\
\hline Calorías de grasa & $\mathrm{cal} / 100 \mathrm{~g}$ & 59,82 & 39,05 & 107,41 & 56,25 \\
\hline Calorías totales & $\mathrm{cal} / 100 \mathrm{~g}$ & 395,13 & 21,67 & 415,41 & 231,53 \\
\hline Fibra dietaria insoluble & $\mathrm{g} / 100 \mathrm{~g}$ & 8.66 & 2,70 & 8,8 & N/A \\
\hline Fibra dietaria soluble & $\mathrm{g} / 100 \mathrm{~g}$ & 3,49 & 0,75 & 3,64 & N/A \\
\hline Fibra dietaria total & $\mathrm{g} / 100 \mathrm{~g}$ & 12,15 & 1,95 & 12,44 & 10,53 \\
\hline
\end{tabular}

1. Harina de chontaduro sin cáscara, promedio de MARCA A y MARCA B comercializada en el Pacífico colombiano, analizada para Corporación Biotec

2. Harina de chontaduro sin cáscara, Desviación Estándar MARCA A y MARCA B comercializada en el Pacífico colombiano, analizada para Corporación Biotec

3. Harina de chontaduro con cáscara elaborada por el proyecto y analizada para Corporación Biotec

4. Cáscara de chontaduro cocido comercializada en el Pacífico colombiano, analizado para Corporación Biotec 
Se puede notar que la PECPC es muy parecida a las harinas de pulpa de chontaduro. El análisis en base seca muestra que contiene un poco más de fibra, con reducción en la concentración de proteínas y grasas.

Esta materia prima es altamente perecible, por tal razón el proceso debe estar en la misma instalación de la industria que lo genera (industria primaria en la cadena de valor) para garantizar que el material no esté sometido a demoras en su procesamiento. Los análisis microbiológicos de metales pesados y plaguicidas realizados a la PECPC no presentaron incumplimientos por fuera de los límites establecidos para el consumo humano por el Codex Alimentarius y la legislación vigente en Colombia. De la misma manera, las aguas residuales de lavado y cocción del chontaduro se presentan como de tipo domésticas, que se pueden tratar con un sistema básico de saneamiento.

\section{El Proceso}

La descripción del proceso con las diferentes etapas que la conforman aparece en la Figura 4.

Figura 4

Diagrama de bloques del proceso de producción de pasta emulsionada de cáscara y pulpa de chontaduro

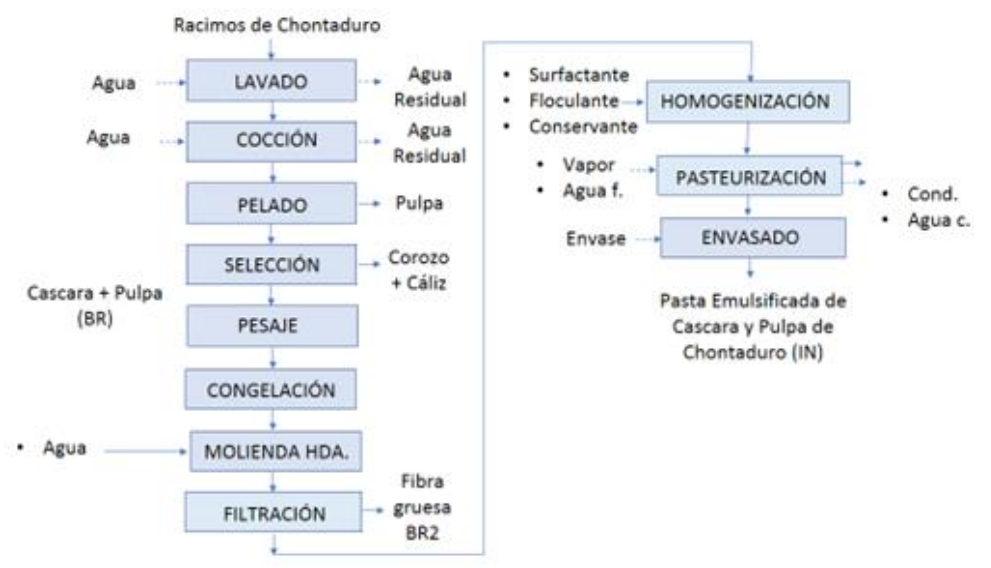

Fuente: Elaboración propia

La Tabla 2 muestra los factores de conversión del proceso.

Tabla 2

Factores de conversión y eficiencias del proceso de producción de pasta emulsionada de cascara y pulpa de chontaduro

\begin{tabular}{|c|c|}
\hline Cultivo & Chontaduro \\
\hline Fruta fresca Kg & 6135 \\
\hline Factor Kg de BR/Kg de fruta & 0,163 \\
\hline Biomasa residual Kg & 1000 \\
\hline Factor Kg IN/ Kg de BR & 1,5 \\
\hline Ingrediente natural (IN) Kg & 1500 \\
\hline Factor Kg BR2/ Kg BR & 0,2 \\
\hline Biomasa residual 2 (BR2) Kg & 200 \\
\hline Eficiencia proceso \% & 88,2 \\
\hline
\end{tabular}

Fuente: Elaboración propia

Puede resumirse el proceso de la siguiente forma: un primer grupo de etapas, que consta de lavado, cocción, pelado, selección, está destinado a la limpieza del chontaduro y a la separación de las fracciones que no cumplan con la calidad de la materia prima que se va a procesar (Moreno, 2005). La cocción permite mejorar la digestibilidad de los carbohidratos contenidos en la pulpa. la Tabla 3.

El resumen de las eficiencias y los factores de conversión obtenidos para el proceso aparece en

Puede constatarse que el proceso es expansivo, es decir, la masa de producto final es superior a la masa de materia prima entrante. La razón para esto es la adición de agua en la etapa de la molienda 
húmeda y la adición de aditivos. Las pérdidas de biomasa residual 2 (fibras largas) no afectan esta expansión de la cantidad de masa que se genera en el producto final.

Tabla 3

Formulación para homogenización de la PECPC

\begin{tabular}{|c|c|c|}
\hline Ingrediente & $\begin{array}{c}\text { Composición } \\
\text { Kg/Kg PECPC }\end{array}$ & $\begin{array}{c}\text { Distribución } \\
\mathbf{\%}\end{array}$ \\
\hline $\begin{array}{c}\text { Cáscara con pulpa adherida de } \\
\text { chontaduro }\end{array}$ & 1 & 74,34 \\
\hline Cutina & 0,25 & 18,58 \\
\hline Goma xanthan & 0,02 & 1,48 \\
\hline Benzoato de sodio & 0,025 & 1,85 \\
\hline Butilhidroxitolueno (BHT) & 0,025 & 1,85 \\
\hline Sorbato de potasio & 0,025 & 1,85 \\
\hline Total & $\mathbf{1 , 3 4 5}$ & $\mathbf{1 0 0}$ \\
\hline
\end{tabular}

Fuente: Elaboración propia

El proceso de producción de la PECPC parte de racimos de chontaduro y no de cáscaras de chontaduro. Ya que se pretende dar elementos de juicio a quien vaya a operar el proceso de las condiciones bajo las cuales se debe hacer el manejo de los racimos recibidos desde el cultivo hasta obtener la materia prima de este proceso, que es la cáscara con pulpa adherida, producto del pelado de los frutos, se mencionan las etapas que esto involucra: lavado, cocción, pelado y selección.

La importancia que esto tiene para la obtención del ingrediente natural radica en que estas operaciones retiran los contaminantes más importantes que pueden provenir del cultivo: materia extraña, plaguicidas, parte de metales pesados y población bacteriana. Si se siguen adecuadamente los procedimientos se supone que se llega con una carga de contaminantes reducida.

La etapa de congelación facilita la molienda posterior y, además, se necesita por logística para mantener un inventario de materia prima para periodos cortos de ausencia de envíos. Su justificación es la logística de manejo de los materiales. Debe ser congelada para evitar proliferación de microorganismos.

La etapa de molienda húmeda permite reducir de tamaño las cáscaras de chontaduro, dando uniformidad a estas. Esta operación se realiza agregando agua y las cáscaras de chontaduro en una licuadora. Se garantiza un tamaño de partícula media de $700 \mu \mathrm{m}$. Esto reduce la longitud de la fibra de la cáscara. Se produce en promedio $1,6 \mathrm{Kg}$ de molienda húmeda de cáscara de chontaduro por cada $\mathrm{Kg}$ de biomasa residual (cáscara de chontaduro) que ingresa al proceso de licuado.

La etapa de filtración retira del proceso aquellas fibras largas por medio de una filtración con malla de acero inoxidable de $7 \mathrm{~mm}$, las cuales van a constituir el mayor volumen de residuo que genera el proceso y cuyo destino debe determinarse a escala real, por ejemplo, entre lo más recomendable para la alimentación de rumiantes o la producción de compostaje.

La homogenización se llevó a cabo en un molino coloidal, el cual realiza una molienda muy fina de las partículas procedentes de las cáscaras de chontaduro hasta un tamaño medio de partícula de $41 \mu \mathrm{m}$. Posteriormente, con la ayuda de los aditivos se promueve la emulsión de los aceites presentes en el chontaduro con las fracciones acuosas de la molienda húmeda para formar la pasta, en la misma operación (Friberg y Kayali, 1991; Robins y Wilde, 2003). Esto garantiza que todo el contenido del chontaduro, con sus sabores y olores, va a estar presente en una suspensión coloidal estable.

Los aditivos usados fueron cutina (agente emulsificante para aceites, grasas, disolventes) y ceras, que proporcionan emulsiones $\mathrm{O} / \mathrm{W}$, benzoato de sodio y sorbato de potasio, útiles como conservantes en la mayoría de los alimentos. Estos se usaron, pues inhiben la actividad microbiológica de levaduras, bacterias y mohos. BHT es un compuesto químico que retarda la rancidez de grasas, aceites vegetales y animales, así como de los alimentos que los contienen y de goma xantha, cuya 
función es la de actuar como coloide hidrofílico para espesar, suspender y estabilizar emulsiones y otros sistemas basados en agua. Fácilmente soluble en agua caliente o fría, proporciona viscosidad estable de las soluciones en amplios rangos de temperatura; son aditivos ampliamente utilizados en la industria alimentaria y que aseguran la conservación de la calidad e inocuidad de la PECPC.

La etapa de pasteurización se realizó a $90{ }^{\circ} \mathrm{C}$, por un tiempo de 30 minutos, hasta bajar la temperatura a $10{ }^{\circ} \mathrm{C}$ por otros 30 minutos, y cumplir con la normatividad existente para un aditivo en alimentos.

Las pérdidas por filtración (biomasa residual 2) son menores que las adiciones. La eficiencia global del proceso, sin considerar perdidas en los equipos por lavados de estos, es de 88,2\%.

\section{Características del Producto Final}

El proceso conserva bastante bien las características nutricionales de la materia prima en el producto final (PECPC). Esto se puede ver en la Tabla 4. Los aditivos usados contribuyen por su carácter aceitoso, que aportan grasas y ácidos grasos, a enriquecer el contenido calórico del ingrediente natural visualizado en la Figura 5.

\section{Figura 5}

Pasta emulsionada de cáscara y pulpa de chontaduro

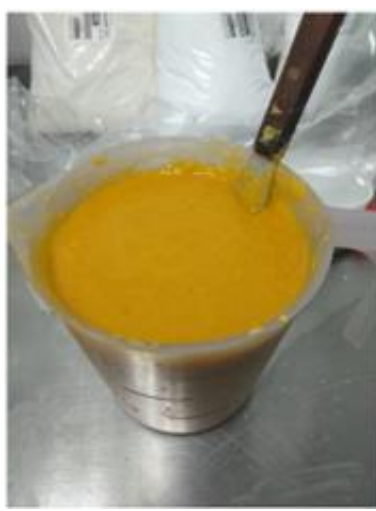

Fuente: Elaboración propia

Tabla 4

Comparación entre la materia prima y el producto final (composición en base húmeda)

\begin{tabular}{|c|c|c|c|c|}
\hline Análisis & Unidad & $\begin{array}{c}\text { Cáscaras } \\
\text { chontaduro }\end{array}$ & $\begin{array}{c}\text { PECPC } \\
\text { promedio }\end{array}$ & $\begin{array}{c}\text { Desviación } \\
\text { estándar }\end{array}$ \\
\hline Carbohidrato & $\mathrm{g} / 100 \mathrm{~g}$ & 39,84 & 12,49 & 2,59 \\
\hline Grasa total & $\mathrm{g} / 100 \mathrm{~g}$ & 6,25 & 6,12 & 0,92 \\
\hline Proteína & $\mathrm{g} / 100 \mathrm{~g}$ & 3,98 & 1,01 & 0,22 \\
\hline Cenizas & $\mathrm{g} / 100 \mathrm{~g}$ & 0,81 & 0,58 & 0,16 \\
\hline Humedad & $\mathrm{g} / 100 \mathrm{~g}$ & 49,12 & 79,8 & 3,53 \\
\hline Total & $\mathrm{g} / 100 \mathrm{~g}$ & $\mathbf{1 0 0}$ & $\mathbf{1 0 0}$ & -- \\
\hline \multicolumn{5}{|c|}{$\mathrm{g}$} \\
\hline Humedad & $\mathrm{g} / 100 \mathrm{~g}$ & 49,12 & 79,8 & 3,53 \\
\hline Sólidos secos & $\mathrm{g} / 100 \mathrm{~g}$ & 50,88 & 20,2 & 3,53 \\
\hline Total & $\mathrm{g} / 100 \mathrm{~g}$ & $\mathbf{1 0 0}$ & $\mathbf{1 0 0}$ & - \\
\hline \multicolumn{5}{|l|}{} \\
\hline Calorías de grasa & $\mathrm{cal} / 100 \mathrm{~g}$ & 56,25 & 55,11 & 8,28 \\
\hline Calorías totales & $\mathrm{cal} / 100 \mathrm{~g}$ & 231,53 & 103,2 & 14,55 \\
\hline \multicolumn{5}{|l|}{} \\
\hline Fibra dietaria total & $\mathrm{g} / 100 \mathrm{~g}$ & 10,53 & 4,04 & 0,73 \\
\hline
\end{tabular}

Fuente: Elaboración propia

\section{Pruebas de Concepto}

Se realizó una prueba de concepto en un panel con 5 jueces entrenados o altamente entrenados, reclutados por agudeza sensorial y motivación, expertos en productos naturales y derivados del chontaduro, usando la PECPC como parte de formulaciones para 1) una salsa 2) el ingrediente solo 3) 
un yogurt 4) una bebida. Los resultados mostraron buenas características físicas. Se notó poco aporte de aroma del chontaduro por la presencia de la PECPC. La mayor debilidad fue un sabor amargo después de ingerirlo, debido probablemente a compuestos como polifenoles, como consecuencia de la falta de un proceso óptimo de desaireación de la pasta. En la Tabla 5 se muestran los resultados de la prueba de concepto realizada a los productos.

Por otro lado, el producto cumple con el tiempo de vida útil estimado para 4 meses en almacenamiento a temperatura de $35 \mathrm{oC} \pm 5 \mathrm{oC}$ y $80 \% \pm 5 \%$ de humedad relativa. Se pueden evaluar posibles mejoras en el material de empaque y en las condiciones de almacenamiento, por ejemplo, ubicando el producto lejos de la luz directa del sol para mantener la calidad sensorial del producto en lugares con temperaturas inferiores a $\operatorname{los} 35 \mathrm{oC} \pm 5 \mathrm{oC}$ y humedad relativa inferior a $80 \% \pm 5 \%$. Es recomendable envasar en un empaque hermético y resistente que aísle el producto de las condiciones ambientales externas; esto favorecerá a la PECPC hasta el final de su vida útil.

Tabla 5

Puntuación prueba de concepto en PECPC en diferentes productos finales

\begin{tabular}{|c|c|c|c|}
\hline Producto & $\begin{array}{c}\text { Calificación } \\
\mathbf{\%}\end{array}$ & Valor máximo & Valor mínimo \\
\hline Pasta & 86,7 & Textura/A. visual & Sabor/Sabor residual \\
\hline Salsa & 81,9 & Textura/A. visual & Aroma/Sabor residual \\
\hline Yogurt & 72,9 & A. visual & Sabor residual \\
\hline Bebida & 72,4 & A. visual & Sabor residual \\
\hline
\end{tabular}

Fuente: Elaboración propia

\section{Conclusiones}

De todo lo expuesto anteriormente, se puede concluir que el ingrediente natural PECPC da una calificación entre media y buena para los productos con los que se combinó en la prueba con usuarios. El sabor residual amargo y picante es crítico en la apreciación de los sabores. La textura y la apariencia visual parecen ser los aspectos más destacados. Es necesaria la posterior modificación de las formulaciones para hacer más aceptable el producto por parte de los consumidores.

Sería ideal contar con un proceso en planta a temperaturas ambiente menores a $20^{\circ} \mathrm{C}$ para mantener la población de mesófilos aerobios en el menor número posible y alargar la vida útil del IN. Es pertinente analizar la dilución que se presenta por ser un proceso expansivo, si es conveniente usar mayor cantidad de cáscara y pulpa de chontaduro o si se incluye la fibra que se retira por filtración, esto último con el objetivo de evitar la pérdida de compuestos nutricionales en el producto final. No sería recomendable almacenar por tiempos prolongados el producto, ya que esto favorece la proliferación de microrganismos del tipo mesófilos.

Finalmente, el producto presentado es una alternativa viable para aprovechamiento de subproductos del chontaduro o biomasa residual resultante del proceso de pelado del chontaduro, aprovechando su composición nutricional, con presencia de ácidos grasos, fibra dietaria y proteína. Es necesario establecer ciclos de prototipación posteriores para ajustar las condiciones de operación y conservar mejor los nutrientes, como carotenoides presentes en la materia prima, los cuales se degradan con mayor facilidad debido a los procesos de calentamiento y pasteurización.

Las cualidades que se le atribuyen a este producto derivado del chontaduro parten de la composición libre de gluten, colesterol, grasas trans y azúcares, apto para personas con enfermedad celíaca. Además de ser buena fuente de fibra para el mejor funcionamiento del sistema digestivo. El uso de dietas bajas en grasa saturada, colesterol y ricas en frutas, verduras, leguminosas y cereales que contienen fibra dietaria, pueden ayudar a reducir el riesgo de enfermedad cardiovascular.

Por último, posee características que le confieren los ácidos grasos omega 3,6 y 9 (FernándezPiedra et al., 1995). Estas dietas ricas en ácidos grasos insaturados, bajas en grasa saturada y colesterol y ricas en frutas, verduras, leguminosas y cereales que contienen fibra dietaria reduce el riesgo de enfermedad cardiovascular. Es además un alimento de bajo índice glicémico (Jatunov et al., 2010; 
Quesada et al., 2011), con carbohidratos complejos de absorción lenta que no favorecen la rápida elevación del azúcar en la sangre.

\section{Reconocimientos}

El presente trabajo recoge los resultados sobre la obtención de PECPC que hace parte de la investigación denominada "Fortalecimiento de las capacidades de $\mathrm{I}+\mathrm{D}+\mathrm{i}$ para la producción de ingredientes naturales a partir de biomasa residual, Palmira, Valle del Cauca, Occidente" (Ortiz et al., 2019), a cargo de Corporación Biotec y la Universidad del Valle.

\section{Referencias}

Reyes, R., Peña, E., \& Gómez, J. (2002). El cultivo de Chontaduro (Bactris gasipaes K.) para palmito. Manual Técnico No. 4. Corporación colombiana de investigación agropecuaria - AGROSAVIA COLCIENCIAS

Hernández, J., Mora, J., \& Rocha, U. (2008). Diversidad genética y relaciones de parentesco de las poblaciones silvestres y cultivadas de pejibaye (Bactris gasipaes, Palmae), utilizando marcadores microsatelitales. Revista de Biología Tropical, 56(1) 21-45, DOI: 10.15517/rbt.v56i1.5520.

Giraldo, A., Dufour, D., Rivera, A., Sánchez, T., Scheldeman X., \& Gonzáles, A. (1999). Estudio de la diversidad del Chontaduro (Bactris Bactris gasipaes gasipaes) consumido en Colombia. CIAT, Centro Internacional de Agricultura Tropical, Cali, Colombia, CIRAD, Centre de Coopération Internationale en Recherche Agronomique pour le Développement, Montpellier, Francia, Bioversity International, Cali, Colombia

López-Calvo, R., Pérez, A., Ivankovich, C., Villaplana, S., \& Castro, M. (2015). Evaluación de la aceptación por consumidores de un bocadillo de pejibaye (Bactris gasipaes) y estudio de su potencial como alimento funcional. Arch. Latinoam. Nutr., 65(1), 51-58.

Martínez, J., \& Ordóñez, L. (2016). Determinación de la concentración de pigmentos carotenoides en harina de residuos de chontaduro (Bactris gasipaes). Prod. + Limpia, 11(1), 85-93. DOI: 10.22507/pml.v11n1a8.

Martínez-Girón, J., Rodríguez-Rodríguez, X., Pinzón-Zárate, X., \& Ordóñez-Santos, L. (2017). Caracterización fisicoquímica de harina de residuos del fruto de chontaduro (Bactris gasipaes Kunth, Arecaceae) obtenida por secado convectivo. Corpoica Cienc. y Tecnol. Agropecu., 18(3), 599-613. DOI: 10.21930/rcta.vol18_num3_art:747.

Ordoñez-Santos, L., Martínez-Girón, J., \& Villamizar-Vargas, R. (2018). Encapsulation of $\beta$-carotene extracted from peach palm residues: A stability study using two spray-dried processes. DYNA, 85(206), 128-134. DOI: $10.15446 /$ dyna.v85n206.68089.

Girón, J., Ordoñez, L., \& Rodríguez-Rodríguez, D. (2019). Extracción de carotenoides totales a partir de epicarpio de chontaduro (Bactris gasipaes) mediante aplicación de ultrasonido y aceite vegetal/Extraction of total carotenoids from peach palm fruit (Bactris gasipaes) peel by means of ultrasound application. Dyna; Bogota, 86 (209), 91-96.

http://search.proquest.com/docview/2244650081/abstract/C78A1126CF5C47FCPQ/1\%0Ahttp://files/6 36/Girón. Extracción de carotenoides totales a partir de epi.pdf.

Sam, G., \& Vásquez, O. (2002). HARINA DE LOS SUBPRODUCTOS DE Bactris gasipaes HBK Y SU USO EN PANIFICACIÓN. Rev. Amaz. Investig. Aliment., vol. 2, núm. 1, pp. 39-47.

Restrepo J., \& J. Estupiñán, J. (2011). Potencial del Chontaduro como Fuente Alimenticia de Alto Valor Nutricional en Países Tropicales. Rev. Ciencias, vol. 11, pp. 1-8. DOI: 10.25100/rc.v11i0.529. 
Rojas-Garbanzo, C., Pérez, A., Bustos-Carmona, J., \& Vaillant, F. (2011). Identification and quantification of carotenoids by HPLC-DAD during the process of peach palm (Bactris gasipaes H.B.K.) flour. Food Res. Int., vol. 44, núm. 7, pp. 23-84. DOI: 10.1016/j.foodres.2011.02.045.

Murillo, M., Kroneberg A., Mata, J., Calzada J., \& Castro, V. (2016). Estudio preliminar sobre factores inhibidores de enzimas proteolíticas en la harina de pejibaye (Bactris gasipaes). Rev. Biol. Trop., vol. 31, núm. 2, pp. 227-231. DOI: 10.15517/rbt.v31i2.24985.

Ministerio de Ambiente y Desarrollo Sostenible (2010). Resolución 0883 de 2018, pp. 2-13.

Ministerio de Ambiente y Desarrollo Sostenible (2015). Resolución 0631 de 2015, pp. 2-22.

Ministerio De Salud y Protección Social (2013). Resolución 3929 De 2013, Ministerio De Salud y Protección Social, pp. 25-27.

Ministerio de Salud y Protección Social (2013). Resolución Número 4506 de 2013, pp. 2-10.

FAO/WHO - Food and Agriculture Organization of the United Nations / World Health Organization (2019). Norma general para los contaminantes y las toxinas presentes en los alimentos y piensos, pp. 75-84.

World Health Organization (2010). The Who Recommended Classification of Pesticides By Hazard and Guidelines To Classification 2009.

Ministerio de Agricultura y Desarrollo Rural y de la Protección Social (2007). Resolución 2906 de 2007, pp. 249.

Graefe, S., Dufour, D., Van Zonneveld, M., Rodriguez, F., \& Gonzalez, A. (2013). Peach palm (Bactris gasipaes) in tropical Latin America: Implications for biodiversity conservation, natural resource management and human nutrition. Biodivers. Conserv., vol. 22, núm. 2, pp. 269-300. DOI: 10.1007/s10531-012-0402-3.

Valencia, R., Montúfar, R., Navarrete, H., \& Balslev, H. (2013). Palmas Ecuatorianas: Biología y uso sostenible.

MADR (2017). Reporte: Área, Producción y Rendimiento Nacional por Cultivo. Agronet.

Moreno, L. (2005). Manual técnico de frutas y verduras. ABC de las frutas y verduras.

Friberg, S., \& Kayali, I. (1991). Surfactant Association Structures, Microemulsions, and Emulsions in Foods.

Robins, M., \& Wilde, P. (2003). COLLOIDS AND EMULSIONS. Encyclopedia of Food Sciences and Nutrition, pp. 1517-1524.

Fernández-Piedra, M., Blanco-Metzler, A., \& Mora-Urpí, J. (1995). Contenido de ácidos grasos en cuatro poblaciones de pejibaye, Bactris gasipaes (Palmae). Rev. Biol. Trop., pp. 61-66.

Jatunov, S., Quesada, S., Díaz, C., \& Murillo, E. (2010). Carotenoid composition and antioxidant activity of the raw and boiled fruit mesocarp of six varieties of Bactris gasipaes. Arch. Latinoam. Nutr., vol. 60, núm. 1, p. 99.

Quesada, S., Azofeifa, G., Jatunov, S., Jiménez, G., Navarro, L., \& Gómez, G. (2011). Carotenoids composition, antioxidant activity and glycemic index of two varieties of Bactris gasipaes.

Ortiz, A. (2019). Ejercicio de Vigilancia e inteligencia estratégica para el ingrediente natural: polvo de la cáscara y pulpa de chontaduro. Fortalecimiento de las capacidades de I+D+I para la producción de ingredientes naturales a partir de la biomasa residual Palmira Valle del Cauca, Occidente. pp. 6-81, feb. 25. DOI: 10.1088/1751-8113/44/8/085201. 